\title{
PAPER
}

\section{Molecular electronic decoherence following attosecond photoionisation}

To cite this article: Caroline Arnold et al 2020 J. Phys. B: At. Mol. Opt. Phys. 53164006

View the article online for updates and enhancements.

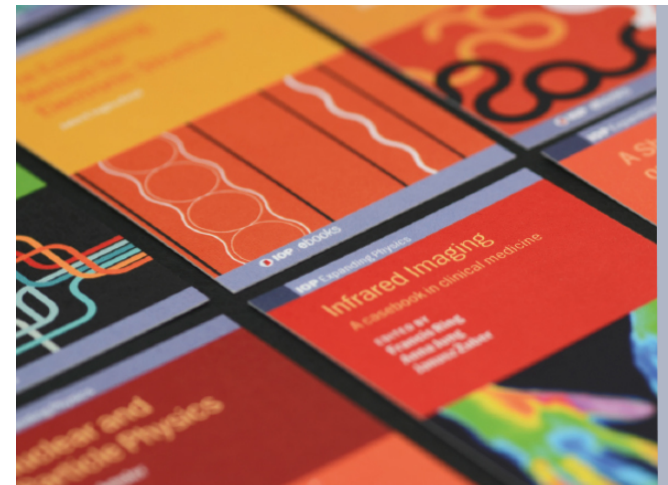

IOP ebooks

Bringing together innovative digital publishing with leading authors from the global scientific community. Start exploring the collection-download the first chapter of every title for free. 


\title{
Molecular electronic decoherence following attosecond photoionisation
}

\author{
Caroline Arnold ${ }^{1,2,3}\left(\mathbb{0}\right.$, Céline Larivière-Loiselle ${ }^{1,4}$, Khadijeh Khalili $^{5}$, \\ Ludger Inhester ${ }^{1,3}{ }^{\circ}$, Ralph Welsch ${ }^{1,3}$ and Robin Santra $^{1,2,3,6}$ a \\ ${ }^{1}$ Center for Free-Electron Laser Science, DESY, Notkestrasse 85, 22607 Hamburg, Germany \\ 2 Department of Physics, Universität Hamburg, Jungiusstrasse 9, 20355 Hamburg, Germany \\ 3 The Hamburg Centre for Ultrafast Imaging, Luruper Chaussee 149, 22761 Hamburg, Germany \\ ${ }^{4}$ Département de Physique, Université de Sherbrooke, J1K 2R1 Sherbrooke, Canada \\ 5 Department of Energy Conversion and Storage, Technical University of Denmark, Anker Egelunds Vej, \\ 2800 Kgs. Lyngby, Denmark \\ E-mail: robin.santra@cfel.de
}

Received 31 January 2020, revised 19 April 2020

Accepted for publication 26 May 2020

Published 30 June 2020

\begin{abstract}
Attosecond pulses can be used to generate coherent superpositions of cationic electronic states in molecules through photoionisation. These can drive coherent electronic dynamics, which may decay within a few femtoseconds due to nuclear motion. In this work, we study the impact of the photoelectron on decoherence in the valence electron system of molecules following attosecond photoionisation. To this end, we include the photoelectron as a classical point charge in a quantum-classical simulation of light-induced ultrafast molecular dynamics and consider ionisation by sub-femtosecond pulses with distinct qualities. By disentangling the contributions of photoelectron and nuclei to the overall electronic decoherence, we find that the photoelectron causes partial decoherence within the first 50 attoseconds. This timescale is noticed to be independent of the ionising pulse. Full electronic decoherence is only seen when the spatial extension of the nuclear wave packet is considered.
\end{abstract}

Keywords: ultrafast molecular dynamics, electronic coherence, attosecond photoionisation, surface hopping

(Some figures may appear in colour only in the online journal)

\section{Introduction}

Attosecond science was established two decades ago with the advent of sub-femtosecond pulses [1,2]. Since then, ultrashort pulses have been employed in numerous applications in fundamental research, from the motion of electrons in atoms [3, 4] to molecules [5, 6] and condensed matter [7, 8].

One particularly interesting field is ultrafast charge dynamics in molecules, a phenomenon at the centre of fundamental biological and chemical processes [9-11]. Charge dynamics can be triggered through photoionisation with coherent attosecond, broadband pulses, where coherent superpositions of electronic states of the remaining cation are created [12-14]. However, photoionisation also gives rise to nuclear dynamics that may destroy valence electronic

\footnotetext{
${ }^{6}$ Author to whom any correspondence should be addressed
}

coherence within a few femtoseconds [15-17]. Electronic decoherence is here defined as the process wherein an initially coherent superposition of electronic states evolves towards a statistical mixture and, as a consequence, coherent electron dynamics are suppressed [18].

The dynamics of coherent electronic superpositions in molecular cations following attosecond photoionisation have been described with various theoretical models. In a widely used approach, nuclear motion is neglected [12, 19]. This defines a purely electronic problem and typically predicts long-lived valence electron dynamics driven by electronic correlations. In order to describe ultrafast electron dynamics in molecules faithfully, it is necessary, however, to take electronic decoherence caused by the nuclei into account. Dephasing effects due to the width of the nuclear wave packet and decrease of coherence due to diminishing wave packet overlap 
cannot be captured otherwise and generally lead to electronic decoherence in the subsystem of valence electrons within a few femtoseconds $[15,20]$.

To incorporate the nuclei, quantum-classical studies were conducted based on sampling of nuclear geometries across the spatial extension of the nuclear wave packet [16, 21]. They show fast dephasing of electronic oscillations with moleculedependent decoherence rates in the range of a few femtoseconds. A full adiabatic quantum-dynamical description of nuclear dynamics following attosecond photoionisation was the focus of our earlier work [15]. This treatment is limited to short-time dynamics, as on-the-fly evaluation of potential energy surfaces is numerically costly for large molecules and, therefore, fitted surfaces are employed. Also within this model, valence electronic decoherence was observed to take place within a few femtoseconds.

It may still be possible to observe coherent electron dynamics close to conical intersections [22], in specially engineered molecules [23] or in small molecules with effectively few nuclear degrees of freedom, e.g. in iodoacetylene [24] or propriolic acid [25]. For a current review of techniques to describe attosecond light-matter interaction and the subsequent electron and nuclear dynamics, see reference [26].

In the works discussed so far, the impact of the photoelectron on electronic coherence is typically neglected, and a fully coherent initial state is assumed. Pabst et al studied the effect of the photoelectron on coherent superpositions of electronic states in xenon undergoing attosecond photoionisation [27]. It was found that interchannel coupling of various hole states reduces electronic coherence within the cationic electronic subsystem within less than 100 as. In related work, the photoelectron dynamics were described classically for strongfield phenomena like high-harmonic generation [28] and double photoionisation $[29,30]$. However, this did not include coupling between the photoelectron and the remaining, bound, quantum electrons.

In this manuscript, we propose a description of the photoelectron as a classical point particle that is released through photoionisation. It is included in a quantum-classical description of the nuclei by the well-established fewest switches surface hopping (FSSH) algorithm [31]. Thus, the dynamics of the classical photoelectron, the bound quantum electrons, and the nuclei are all coupled.

We employ this setup to analyse the decoherence of valence electron dynamics in an isolated water cation, $\mathrm{H}_{2} \mathrm{O}^{+}$in the presence of the photoelectron, following attosecond broadband ionisation.

In section 2, the methodology employed in this manuscript is presented. We then turn to analyse electronic decoherence in the molecular cation in section 3.1, before discussing in detail the photoelectron's contribution to electronic decoherence in section 3.2. The dynamics of the photoelectron are analysed in section 4 , and we conclude the discussion in section 5 .

\section{Methods}

We first describe the implementation of the FSSH algorithm in section 2.1. Then we turn towards the sampling of electron and nuclear configurations in section 2.2. Electronic coherence is defined in section 2.3.

\subsection{Fewest-switches surface hopping}

The dynamics of the molecular cation and the classical photoelectron are described using the FSSH method [31] with the implementation given in references [32,33]. The classical photoelectron is included as a negatively charged point particle. As in the standard implementation of the FSSH algorithm, the nuclei and the classical photoelectron move on excitedstate potential energy surfaces. Along this trajectory $\vec{R}(t)$, the time-dependent Schrödinger equation is solved for the bound electrons,

$$
i \hbar \dot{c}_{k}(t)=\sum_{j} c_{j}(t)\left[V_{k j}-i \hbar \dot{\vec{R}} \cdot \vec{d}_{k j},\right]
$$

where $c_{j}$ are the expansion coefficients of the electronic wave function, $V_{k j}$ the matrix elements of the electronic Hamiltonian, and $\vec{d}_{k j}$ the non-adiabatic coupling vectors. The matrix $V_{k j}$ is diagonal in the adiabatic representation employed here. The elements of the electronic density matrix are given as

$$
\rho_{j k}=c_{j} c_{k}^{*}
$$

The rate of change of the population of the $j$ th electronic state is

$$
\dot{\rho}_{j j}=\sum_{k \neq j} b_{k j}, \quad b_{k j}=-2 \operatorname{Re}\left[\rho_{j k} \dot{\vec{R}} \cdot \vec{d}_{k j}\right] .
$$

Non-adiabatic transitions, i.e. hops between these surfaces, are possible when the surfaces come close in energy. The switching probability from state $j$ to $k$ is given as

$$
P_{j \rightarrow k}=-\frac{b_{k j} \Delta t}{\rho_{j j}},
$$

with the trajectory time step $\Delta t$. The switching probability is compared to a uniform random number $\zeta, 0<\zeta<1$, and if $P_{j \rightarrow k}>\zeta$, the hop occurs. For energy conservation across the gap between the participating potential energy surfaces, the velocities of the classical trajectory are then adjusted in the direction of the non-adiabatic coupling vector. We employ our in-house electronic structure toolkit XMOLECULE [34] to retrieve the excited-state gradients and non-adiabatic coupling matrix elements that are required for FSSH. These quantities are evaluated at the Hartree-Fock level of theory, which enables us to use Koopmans' theorem for excited cationic states $[35,36]$. All three subsystems, the nuclei, the bound quantum electrons, and the classical point-like photoelectron are electrostatically coupled.

We employ the $6-31+\mathrm{G}$ basis set, which includes diffuse basis functions for the oxygen atom [37], and a simulation time step of 1 as. Close encounters of the classical photoelectron and the positively charged nuclei lead to numerical instability, resulting in an unphysical loss of energy along the 


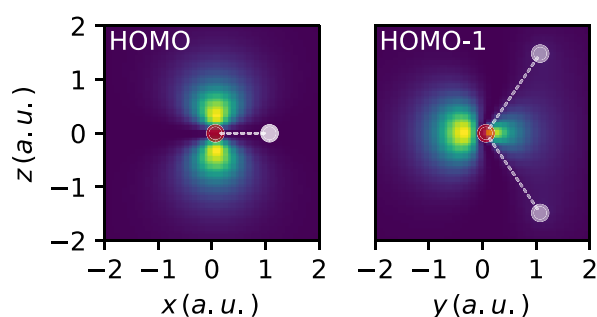

Figure 1. Molecular orbital density of the $1 b_{1}$ orbital (HOMO, left) and $3 \mathrm{a}_{1}$ orbital (HOMO-1, right) of $\mathrm{H}_{2} \mathrm{O}$. The nuclei are included for reference.

trajectory. Therefore, the soft-core correction is introduced to the Coulomb potential [38]:

$$
V(r)=-\frac{Z}{r+\Delta r}
$$

where $Z$ is the atomic number. From the requirement of energy conservation along the trajectory, we determine $\Delta r=$ 0.33a.u. $\approx 0.17 \AA$ for the water monomer.

Note that FSSH is known for overestimating electronic coherence [39]. This has been corrected in the augmentedFSSH algorithm established by Subotnik et al [40-42]. Including the correction would lead to an increase in computational complexity that would limit the phase space sampling of configurations for the nuclei. For the more qualitative discussion in the present work, we elect to use standard FSSH.

\subsection{Sampling in phase space}

The initial configurations of both the classical photoelectron and the nuclei in phase space are obtained by statistical sampling. For the nuclei, Wigner sampling is applied [43]. The initial positions of the photoelectron are gathered by rejection sampling from the density of the respective molecular orbitals involved in photoionisation (figure 1). At a given initial position $\vec{r}$, energy conservation requires

$$
\varepsilon_{\mathrm{MO}}+\omega=T(\vec{r})+V_{\mathrm{C}}(\vec{r}),
$$

where $\varepsilon_{\mathrm{MO}}$ is the molecular orbital energy, $\omega$ is the photon energy, and $T(\vec{r})$ and $V_{\mathrm{C}}(\vec{r})$ correspond to the kinetic and (Coulomb) potential energy, respectively. Note that atomic units are employed throughout, thus the initial velocity of the classical electron is given as $v_{\mathrm{el}}=\sqrt{2 T}$. We assume uniform initial distribution for the direction of the velocity vector of the classical photoelectron.

\subsection{Definition of electronic coherence}

Following references $[15,16]$, we define electronic coherence via the reduced density matrix of the electronic subsystem of the remaining, bound (quantum) electrons. As introduced in section 2.1, the quantum state vector is expressed in the basis of adiabatic electronic states $\left\{\left|\phi_{i}\right\rangle\right\}$ as

$$
|\psi\rangle=\sum_{i} c_{i}\left|\phi_{i}\right\rangle
$$

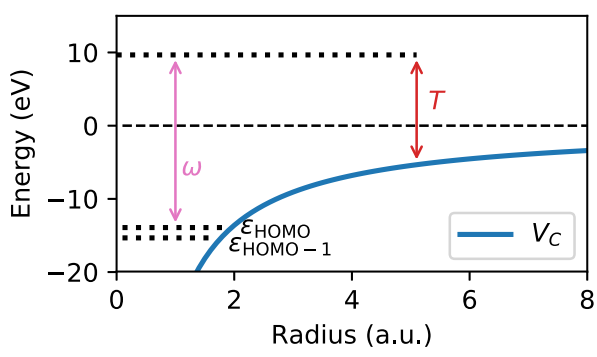

Figure 2. Energy conservation throughout photoionisation. The electron is removed from an orbital with binding energy $\varepsilon_{\mathrm{MO}}$ and experiences a position-dependent Coulomb potential of the positively charged cation, $V_{\mathrm{C}}(r)$. The available kinetic energy is determined by considering the energy that is put in by the ionising photon, see equation (6).

The electronic density matrix is calculated for each classical trajectory, and then averaged [44]:

$$
\rho^{(j)}(t)=\left(\begin{array}{ll}
c_{1}^{*} c_{1} & c_{1} c_{2}^{*} \\
c_{2} c_{1}^{*} & c_{2}^{*} c_{2}
\end{array}\right), \quad \rho=\frac{1}{N} \sum_{j=1}^{N} \rho^{(j)},
$$

where $N$ is the number of classical trajectories.

FSSH is often used to describe problems in photochemistry, with only one initially non-zero coefficient $c_{i}$ [31]. Here, we initially populate a coherent superposition of the two lowestlying electronic states of the remaining cation, corresponding to ionisation either from the HOMO or the HOMO-1. Therefore, the initial coefficient vector has to contain coherences and is set to $c_{1}=c_{2}=1 / \sqrt{2}$. This assumes a coherent ionising pulse that is spectrally broad enough to span both of these energy levels. For the water molecule in the gas phase, they are located within our electronic structure calculations at ionisation potentials of $13.92 \mathrm{eV}$ and $15.33 \mathrm{eV}$, respectively.

The degree of electronic coherence in the initially populated subsystem of excited electronic states is given as [4]

$$
g_{12}=\frac{\left|\rho_{12}\right|}{\sqrt{\rho_{11} \rho_{22}}},
$$

where $g_{12}=1$ corresponds to a fully coherent superposition and $g_{12}=0$ to a completely incoherent mixture. We find that throughout all trajectories, population transfer to higher excited electronic states is negligible (less than 5\%), and therefore the restriction of determining electronic coherence in the subsystem of the two energetically lowest-lying cationic states is reasonable.

\subsection{Attosecond pulses}

For the purpose of this work, we consider instantaneous, vertical photoionisation. The photon energy merely defines the amount of asymptotic kinetic energy available for the photoelectron (cf equation (6)), the pulse intensity does not influence the initial conditions for the electrons.

For the following study, we consider four attosecond pulses with distinct qualities, based on simplified versions of attosecond pulses that have been employed in ultrafast experiments (figure 2). First, we consider pulses whose spectra span about 
Table 1. Range of the spectra of the attosecond pulses considered.

\begin{tabular}{lccc}
\hline Pulse & $\omega_{\min }(\mathrm{eV})$ & $\omega_{\max }(\mathrm{eV})$ & Reference \\
\hline P 1 & 2 & 14 & {$[45]$} \\
P 2 & 14 & 30 & {$[12]$} \\
P 3 & 66 & 146 & {$[46]$} \\
P 4 & 66 & 418 & w/o reference \\
\hline
\end{tabular}

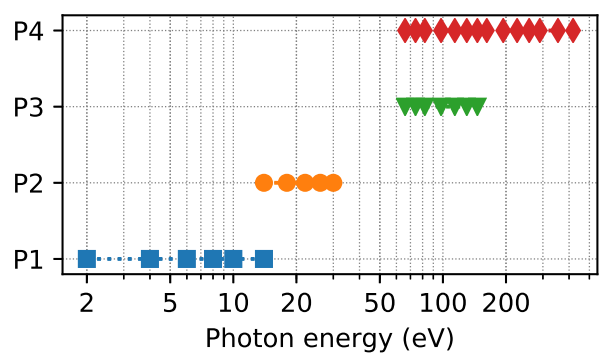

Figure 3. Photon energies considered for the selected attosecond pulses (P $1 \ldots 4$, see table 1 for numerical values). The discrete photon energies actually employed in the simulations are indicated with markers. Note that the values are displayed on a logarithmic scale to enhance the visibility of the low-energy pulses.

$10 \mathrm{eV}$ in bandwidth and are centred at comparatively small XUV photon energies $[12,45]$. Then, we consider a pulse with larger central energy $(100 \mathrm{eV})$ and ultra-broad bandwidth of about $100 \mathrm{eV}$ [46]. A last pulse, not realized yet in experiments, extends up to the soft x-ray region of the electromagnetic spectrum. The respective energy ranges and publications that motivated our choice are summarized in table 1 and displayed in figure 3. For simplicity, we put the same weight on each spectral component of the attosecond pulse and sample discrete photon energies across the spectrum. Thus, the pulse intensity is uniform across the pulse spectrum.

The pulse-dependent overall degree of electronic coherence is determined by averaging over the weighted $g_{12}^{(j)}$ of trajectory ensembles originating from the same photon energy:

$$
\left\langle g_{12}(t)\right\rangle=\frac{1}{M} \sum_{j=1}^{M} g_{12}^{(j)}(t),
$$

where $M$ is the number of photon energies sampled.

\section{Results}

\subsection{Electronic decoherence in an isolated cation}

Figure 4 shows the time evolution of the electronic coherence in the water cation, neglecting the photoelectron. We average over 500 different initial configurations of the nuclei obtained from Wigner sampling and compare these to an adiabatic quantum-dynamical calculation performed with the multi-configuration time-dependent Hartree Method (MCTDH, [47]) in previous work [15]. Due to the complexity of full quantum-dynamical calculations, especially for larger

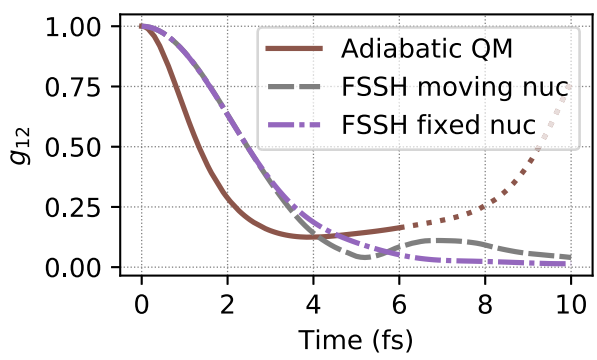

Figure 4. Electronic coherence in the water cation. Results from a full adiabatic quantum-dynamical calculation are displayed. Here, the dotted line indicates unphysical coherence revivals due to limitations of the model (cf reference [15]). Additionally, results from FSSH calculations from 500 Wigner-sampled initial nuclear configurations are shown, both for trajectories with moving nuclei and trajectories with fixed nuclei.

molecules, this type of calculation is only possible on fitted surfaces. This limitation gives rise to the unphysical revival of electronic coherence indicated by the dotted line in figure 4 .

Both models show ultrafast electronic decoherence within a few femtoseconds. However, FSSH underestimates the speed of decoherence by about a factor of 2 . Since we do not employ any correction for decoherence in the FSSH algorithm (cf section 2.1), this is to be expected. FSSH shows a small revival between 5 and $8 \mathrm{fs}$. This may be an effect of the small number of nuclear degrees of freedom, as seen in preceding work [15], and will likely be suppressed in larger molecules. For comparison, figure 4 also includes electronic coherence from FSSH trajectories with fixed nuclei, but still taking into consideration nuclear initial-state sampling. Here, only the dephasing of oscillations among points sampled across the nuclear wave packet contributes to decoherence. Note that this is already sufficient to describe decoherence qualitatively.

\subsection{Electronic decoherence including the photoelectron}

In order to disentangle the relative contributions of the photoelectron and the nuclei to the overall decoherence of the electronic superposition in the cation, we consider in figure 5 different levels of sampling, as well as different treatments of electron and nuclear dynamics. In all panels, we refer to the four pulses given in figure 3. Error bars are indicated as shaded areas and are obtained by bootstrap sampling [48].

In panel (A), we show electronic decoherence averaged over FSSH trajectories from 100 initial photoelectron configurations per ionised molecular orbital and photon energy. The water cation is considered at a single geometry and the nuclei are kept fixed throughout the numerical propagation. We observe a very fast decrease of electronic coherence within the first 40 as (see inset). Thereafter, the degree of electronic coherence drops more slowly to values between 0.50 and 0.60 . While the pulses involving small photon energies (P1, P2) lead to lower final coherence values, the effect is not significant given the numerous assumptions and the statistical errorbars. In the first 50 as, high-energy pulses lead to a slightly faster decoherence than low-energy pulses. Here, and in all following panels, we include the benchmark calculation from the 

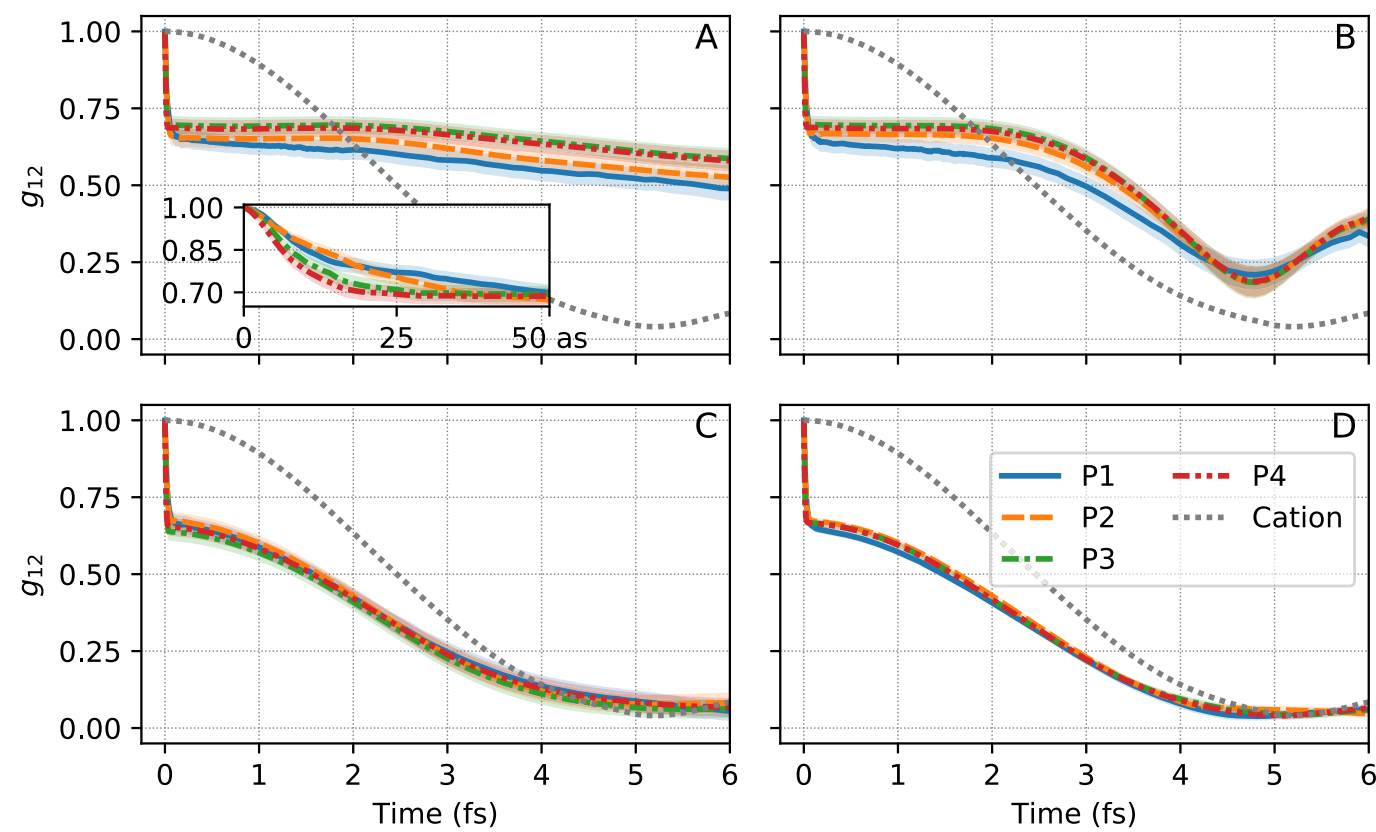

Figure 5. Time evolution of the degree of electronic coherence following attosecond molecular photoionisation by four different pulses (cf figure 3). All panels take into consideration a moving classical photoelectron sampled from the ionised molecular orbital. For reference, the degree of coherence arising from a simulation including only the $\mathrm{H}_{2} \mathrm{O}^{+}$cation (cf figure 4) is included in all panels. The nuclei are incorporated in different ways: (A) fixed nuclei at a single geometry. (B) Moving nuclei, starting from a single geometry. (C) Fixed nuclei sampled at different initial geometries. (D) Moving nuclei sampled at different initial geometries. The error bars are indicated by the shaded areas and are obtained by bootstrap sampling [48].

nuclei-only trajectories (figure 4). The short-term evolution of electronic coherences shown in the inset of panel (A) is found to be similar throughout all following scenarios. We observe that the photoelectron dynamics on their own do not account for the full decoherence seen by the benchmark calculation.

In panel (B), we apply the same sampling as in the preceding panel, but allow the nuclei to move from the geometry used in panel (A). We observe the same sharp drop-off of electronic coherence, followed by a decay and revival. The nuclei move differently on the excited-state surfaces originating from orbitals HOMO or HOMO-1. Therefore, this shows the importance of nuclear wave packet overlap for maintaining electronic coherence. Still, the final degree of coherence is larger than in the benchmark calculation.

In panel (C), we consider 60 geometries for the water cation sampled from a Wigner distribution. Per nuclear configuration, ionised molecular orbital, and photon energy, two configurations are sampled for the classical photoelectron. The nuclei are kept fixed throughout the propagation. Here, full electronic decoherence is observed, as in the cation benchmark, but at initially faster decoherence rate.

In panel (D), we consider 60 Wigner-sampled phase-space points for the water cation. For each nuclear configuration, ionised molecular orbital, and photon energy, 10 configurations are sampled for the classical photoelectron. The phase space is fully sampled, and the nuclei are allowed to move. The large number of sampled trajectories decreases the errorbars to about $3 \%$. Following the electron-induced fast dropoff, electronic coherence quickly vanishes completely. After 5 fs, the value matches the reference curve obtained by sampling only nuclear geometries. Compared to panel (C), the additional contribution of moving nuclei to electronic decoherence is not significant within the statistical errorbars. As in figure 4, a small revival of electronic coherence is seen after 5 . Therefore we conclude that, in order to describe electronic coherence faithfully on the timescale of a few femtoseconds, it is of utmost importance to sample nuclear positions within the initial extension of the nuclear wave packet.

\section{Dynamics of the classical photoelectron}

In this section, we present further analysis of the dynamics of the classical photoelectron based on the trajectories that are displayed in panel (D) of figure 5. The Coulomb force between the classical photoelectron and the molecular cation defines a scattering problem, leading to the deflection of the classical photoelectron. Figure 6 shows the mean angle of deflection of the direction of motion of the classical photoelectron, defined by $\hat{v}_{\mathrm{el}}=\vec{v}_{\mathrm{el}} /\left|\vec{v}_{\mathrm{el}}\right|$, and evaluated for the four attosecond pulses introduced in section 2.4. For the first and second pulse, with a spectrum in the range of a few to tens of $\mathrm{eV}$, the deflection is most prominent. For the third and fourth pulse, with high central photon energies, the classical photoelectron is initially so fast as to not be deflected substantially by the molecular cation. The final value of the deflection angle is reached when the classical photoelectron and the cation are sufficiently far apart from each other.

Figure 7 shows the distance that the electron travels with respect to the oxygen nucleus within $1 \mathrm{fs}$. The width of these distributions mirrors the initial bandwidth of the attosecond pulse (see figure 3). 


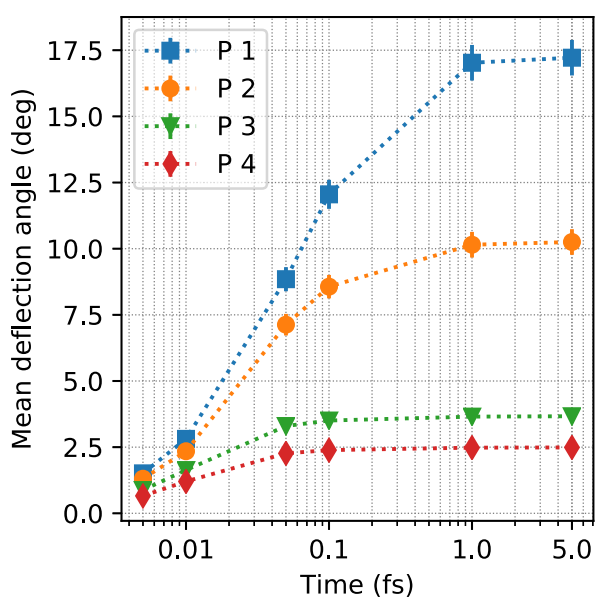

Figure 6. Deflection of the velocity vector of the classical photoelectron as a function of time following instantaneous photoionisation of $\mathrm{H}_{2} \mathrm{O}$. Evaluated for the four attosecond pulses ( $\mathrm{cf}$ figure 3 )

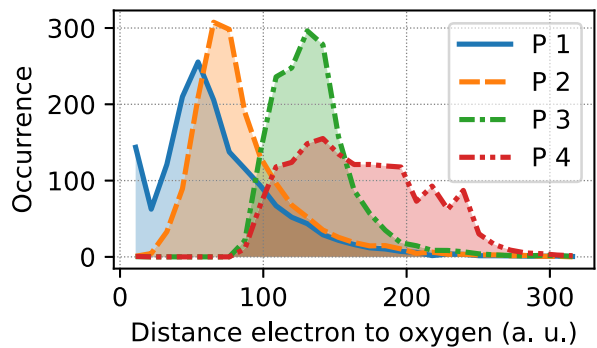

Figure 7. Histogram of the distance of the electron with respect to the oxygen nucleus, taking into account the different ionising pulses. Evaluated after $1 \mathrm{fs}$ and for the four attosecond pulses (cf figure 3).

\section{Conclusion}

We have presented an analysis of attosecond molecular photoionisation of gas phase $\mathrm{H}_{2} \mathrm{O}$ within a simple model of a classical photoelectron, focussing on the time evolution of electronic coherence in the photoion. To this end, we adopted an FSSH description based on Hartree-Fock electronic structure theory that includes the photoelectron as a negatively charged point particle. The spectrum of the attosecond pulse defines the kinetic energy of the released photoelectron, and we considered four pulses of different spectral characteristics.

This setup has allowed us to disentangle the contribution of both photoelectron and nuclei to the overall electronic decoherence in the subsystem of valence electrons. We found that the presence of the classical photoelectron initiates partial decoherence already within 50 as. Full decoherence is only reached when the extension of nuclear geometries is taken into account. Within our model, the effect is not significantly dependent on the spectral range of the ionising attosecond pulse. However, there is a tendency that ionisation with small photon energies leads to less electronic coherence after a few femtoseconds than ionisation with typical XUV photon energies that are the main component of attosecond pulses.

Earlier studies on electron dynamics in the cation follow- ing attosecond photoionisation showed a universal response timescale of 50 as for the rearrangement of the remaining bound electrons $[49,50]$. However, this approach neglected the interaction of the photoelectron and the cation. The results presented here indicate that electronic decoherence, caused by the photoelectron, and correlation-driven electron dynamics in the cation are competing processes on a similar timescale. They serve as a starting point for including the photoelectron in attosecond photoionisation of molecules in future work.

In the current setup, the treatment of larger molecular cations and their photoelectron is numerically costly. A solution for future work may be to adopt different time steps for the classical photoelectron and the heavier nuclei. Such an approach would be especially valuable in order to shed light on the ultrafast dynamics of the photoelectron in liquid water [51], since the hydrated electron is known to influence spectroscopic signals [52]. We recently studied ultrafast hole dynamics in strong-field ionised liquid water leading to the formation of free radicals, including non-Born-Oppenheimer effects [53].

A future quantitative description of the influence of the photoelectron in such an experiment would require decoherence corrections to the FSSH algorithm, as it was seen that the lifetime of the hydrated electron is erroneously short without them [54]. To this end, the augmented FSSH algorithm could be employed [44]. Finally, the quantum nature of the photoelectron could be partially taken into account using ring-polymer molecular dynamics [55-57].

\section{Acknowledgments}

This work was supported in part by the Cluster of Excellence 'CUI: Advanced Imaging of Matter' of the Deutsche Forschungsgemeinschaft (DFG)—EXC 2056-Project ID 390715994. CL-L acknowledges support by the RISE Programme of the DAAD.

\section{ORCID iDs}

Caroline Arnold (D) https://orcid.org/0000-0002-9458-1517

Ludger Inhester (iD https://orcid.org/0000-0003-1417-4151

Ralph Welsch (D) https://orcid.org/0000-0001-9995-7926

Robin Santra (D) https://orcid.org/0000-0002-1442-9815

\section{References}

[1] Hentschel M et al 2001 Nature 414 509-13

[2] Corkum P B and Krausz F 2007 Nat. Phys. 3 381-7

[3] Nisoli M and Sansone G 2009 Prog. Quantum Electron. 33 17-59

[4] Goulielmakis E et al 2010 Nature 466 739-43

[5] Krausz F and Ivanov M 2009 Rev. Mod. Phys. 81 163-234

[6] Nisoli M, Decleva P, Calegari F, Palacios A and Martín F 2017 Chem. Rev. 117 10760-825

[7] Cavalieri A L et al 2007 Nature 449 1029-32

[8] Ramasesha K, Leone S R and Neumark D M 2016 Annu. Rev. Phys. Chem. 67 41-63

[9] Cederbaum L S and Zobeley J 1999 Chem. Phys. Lett. 307 205-10 
[10] Remacle F and Levine R D 2006 Proc. Natl Acad. Sci. 103 6793-8

[11] Wörner H J et al 2017 Struct. Dyn. 4061508

[12] Calegari F et al 2014 Science 346 336-9

[13] Timmers H, Li Z, Shivaram N, Santra R, Vendrell O and Sandhu A 2014 Phys. Rev. Lett. 113113003

[14] Kraus P M and Wörner H J 2013 ChemPhysChem 14 $1445-50$

[15] Arnold C, Vendrell O and Santra R 2017 Phys. Rev. A 95 033425

[16] Vacher M, Steinberg L, Jenkins A J, Bearpark M J and Robb M A 2015 Phys. Rev. A 92040502

[17] Patchkovskii S 2009 Phys. Rev. Lett. 102253602

[18] Zhu C, Nangia S, Jasper A W and Truhlar D G 2004 J. Chem. Phys. 121 7658-70

[19] Lara-Astiaso M et al 2018 J. Phys. Chem. Lett. 9 4570-7

[20] Vacher M, Bearpark M J, Robb M A and Malhado J A P 2017 Phys. Rev. Lett. 118083001

[21] Jenkins A J, Vacher M, Bearpark M J and Robb M A 2016 J. Chem. Phys. 144104110

[22] Arnold C, Vendrell O, Welsch R and Santra R 2018 Phys. Rev. Lett. 120123001

[23] Jenkins A J, Vacher M, Twidale R M, Bearpark M J and Robb M A 2016 J. Chem. Phys. 145164103

[24] Jia D, Manz J and Yang Y 2019 J. Phys. Chem. Lett. 10 4273-7

[25] Despré V, Golubev N V and Kuleff A I 2018 Phys. Rev. Lett. 121203002

[26] Palacios A and Martín F 2020 WIREs Comput. Mol. Sci. 10 1430

[27] Pabst S, Greenman L, Ho P J, Mazziotti D A and Santra R 2011 Phys. Rev. Lett. 106053003

[28] Kamor A, Chandre C, Uzer T and Mauger F 2014 Phys. Rev. Lett. 112133003

[29] Emmanouilidou A 2008 Phys. Rev. A 78023411

[30] Ho P J, Panfili R, Haan S L and Eberly J H 2005 Phys. Rev. Lett. 94093002

[31] Tully J C 1990 J. Chem. Phys. 93 1061-71

[32] Bazzi S, Welsch R, Vendrell O and Santra R 2018 J. Phys. Chem. A 122 1004-10

[33] Ganesa Subramanian M K, Santra R and Welsch R 2018 Phys. Rev. A 982469
[34] Hao Y, Inhester L, Hanasaki K, Son S K and Santra R 2015 Struct. Dyn. 2041707

[35] Khalili K, Inhester L, Arnold C, Welsch R, Andreasen J W and Santra R 2019 Struct. Dyn. 6044102

[36] Li Z, Madjet M E A, Vendrell O and Santra R 2014 Faraday Discuss. 171 457-70

[37] Clark T, Chandrasekhar J, Spitznagel G W and Schleyer P V R 1983 J. Comput. Chem. 4 294-301

[38] Gordon A, Santra R and Kärtner F X 2005 Phys. Rev. A 72 063411

[39] Subotnik J E, Jain A, Landry B, Petit A, Ouyang W and Bellonzi N 2016 Annu. Rev. Phys. Chem. 67 387-417

[40] Subotnik J E and Shenvi N 2011 J. Chem. Phys. 134244114

[41] Subotnik J E and Shenvi N 2011 J. Chem. Phys. 134024105

[42] Shenvi N, Subotnik J E and Yang W 2011 J. Chem. Phys. 135 024101

[43] Wigner E 1932 Phys. Rev. 40 749-59

[44] Landry B R and Subotnik J E 2012 J. Chem. Phys. 137 22A513

[45] Singh K P et al 2010 Phys. Rev. Lett. 104023001

[46] Gaumnitz T, Jain A, Pertot Y, Huppert M, Jordan I, Ardana-Lamas F and Wörner H J 2017 Opt. Express 25 27506

[47] Beck M H, Jäckle A, Worth G A and Meyer H D 2000 Phys. Rep. 324 1-105

[48] Efron B 1979 Ann. Stat. 7 1-26

[49] Breidbach J and Cederbaum L S 2005 Phys. Rev. Lett. 94 033901

[50] Kuleff A I and Cederbaum L S 2014 J. Phys. B: At. Mol. Opt. Phys. 47124002

[51] Nordlund D, Ogasawara H, Bluhm H, Takahashi O, Odelius M, Nagasono M, Pettersson L G M and Nilsson A 2007 Phys. Rev. Lett. 99217406

[52] Turi L and Rossky P J 2012 Chem. Rev. 112 5641-74

[53] Loh Z H 2020 Science 367 179-82

[54] Wong K F and Rossky P J 2002 J. Chem. Phys. 1168418

[55] Craig I R and Manolopoulos D E 2004 J. Chem. Phys. 121 3368-73

[56] Menzeleev A R and Miller T F 2010 J. Chem. Phys. 132 034106

[57] Welsch R, Song K, Shi Q, Althorpe S C and Miller T F 2016 J. Chem. Phys. 145204118 